\title{
Neutrino tomography of the Earth
}

\author{
Jordi Salvado* \\ Departament de Fisíca Quàntica i Astrofísica and Institut de Ciències del Cosmos, Universitat \\ de Barcelona, \\ Diagonal 647, E-08028 Barcelona, Spain \\ E-mail: jsalvado@icc.ub.edu
}

\section{Andrea Donini}

Instituto de Física Corpuscular (IFIC), CSIC-Universitat de València,

E-46071 Valencia, Spain

\section{Sergio Palomares-Ruiz}

Instituto de Física Corpuscular (IFIC), CSIC-Universitat de València,

E-46071 Valencia, Spain

For the first time, a statistically significant tomography of the Earth, using the attenuation of high energy atmospheric neutrinos, has been performed. Here we summarize the results obtained with one year of IceCube data [1]. The density distribution of the Earth is obtained with a five-layer model, confirmed to be statistically compatible with the PREM model. The same averaged density in the core and the mantel is excluded with pvalue $=1 \%$, with preference for a denser core region. The total mass and momentum of inertia are statistically consistent with the gravitational inferred measures.

Neutrino Oscillation Workshop (NOW2018)

9 - 16 September, 2018

Rosa Marina (Ostuni, Brindisi, Italy)

\footnotetext{
* Speaker.
} 


\section{Introduction}

Measuring the density profile of the Earth is essential to solve a number of important problems in geophysics, such as the dynamics of the core and mantle, the mechanism of the geomagnetic dynamo or the bulk composition of the Earth [2]. Most of our knowledge about the internal structure of the Earth and the physical properties of its different layers comes from seismological data.

Neutrinos can be used to study the Earth's interior in several ways [3]. Our knowledge of neutrino propagation through the Earth may give relevant information about the Earth's density profile. Neutrino propagation does depend on the details of the matter structure between the source and the detector. For neutrinos with energies below $1 \mathrm{TeV}$, the matter profile affects the neutrino oscillation pattern, whereas neutrinos with energies in the multi- $\mathrm{TeV}$ range may scatter via chargedcurrent and neutral-current interactions with the nucleons along the path and get absorbed, therefore the neutrino flux observed at the detector depends on the number of nucleons along its path.

The idea of using neutrinos to perform absorption radiographies of the Earth was suggested more than four decades ago [4,5], although the first forecast study with atmospheric neutrinos is only a decade old [6]. Currently, the IceCube neutrino telescope is the largest detector measuring multi- $\mathrm{TeV}$ atmospheric neutrinos, with enough statistics to be sensitive to the absorption effect. Motivated by this, one-year of through-going muon data was considered [1] to perform the first tomography of the Earth using actual neutrino data. The main results are summarized in this talk.

\section{Data and Analysis}

In this work we use the publicly available IceCube one-year up-going muon sample, collected during 2011-2012 and referred, this data was collected with the final configuration of the IceCube detector with 86 strings (IC86). It contains 20145 muons detected over a live time of 343.7 days [7] (a preliminary attempt using IceCube data with very limited event statistics was presented in 2012 [8]). These muons are produced by up-going neutrinos and antineutrinos which, after crossing the Earth, interact via charged-current processes in the bedrock or ice surrounding the detector. The purity of the sample is $\approx 99 \%$, the the zenith angle is between $-1<\cos \theta_{z}<0.2$ and the reconstructed muon energy spans in $400 \mathrm{GeV} \lesssim E_{\mu}^{\text {rec }} \lesssim 20 \mathrm{TeV}$.

The distribution in cosine of the zenith angle of the data relative to the number of expected events without attenuation is shown in fig. 1 for the full energy range and $E_{\mu}^{\text {rec }}>5 \mathrm{TeV}$. The blue band show the 1- $\sigma$ region for the expected measurements assuming the Preliminary Reference Earth Model (PREM).

In order to account for systematic uncertainties, we consider four of the continuous nuisance parameters described in the IC86 paper [7]: The overall flux normalization $(N)$, the pion-to-kaon ratio $(\pi / K)$ determines the relative contribution to the neutrino flux from pion or kaon decays, the uncertainty on the spectral shape of the atmospheric neutrino spectrum, $\Delta \gamma$, is accounted for by a tilt in the energy spectrum, and the uncertainty in the efficiency of the digital optical modules (DOM $\mathrm{Dff})$.

We do a binned maximum likelihood fit for the nuisance parameters and use Multinest [9] to perform a Bayesian inference in $\left(\rho_{1}, \rho_{2}, \rho_{3}, \rho_{4}, \rho_{5}\right)$, that correspond to the densities in the innercore region $\left(\rho_{1}\right)$, two equal-radius layer in the outer core $\left(\rho_{2}, \rho_{2}\right)$, and two equal-radius layers in 
a

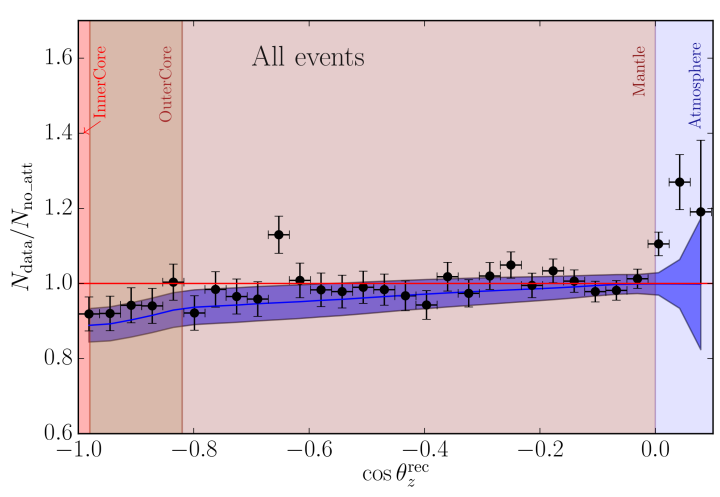

b

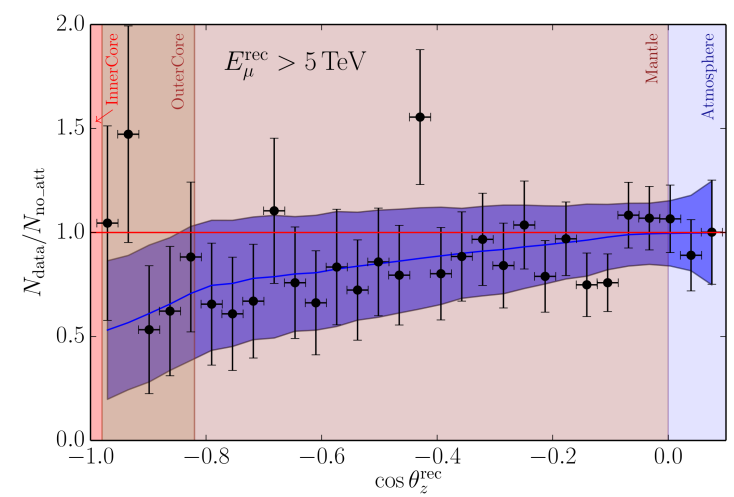

Figure 1: Distribution in cosine of the zenith angle of the data relative to the number of expected events without attenuation: for the full energy range (a), and for $E_{\mu}^{\text {rec }}>5 \mathrm{TeV}$ (b). From ref. [1]

the mantle region $\left(\rho_{4}, \rho_{5}\right)$. We use the likelihood function,

$$
\log \mathscr{L}\left(\rho_{1}, \rho_{2}, \rho_{3}, \rho_{4}, \rho_{5}\right)=\min _{\vec{\theta},\{d\}}\left(\sum_{i=1}^{N_{\text {bins }}}\left[x_{i} \log \lambda_{i}(\vec{\theta})-\lambda_{i}(\vec{\theta})\right]+\sum_{\eta} \frac{\left(\theta_{\eta}-\Theta_{\eta}\right)^{2}}{\sigma_{\eta}^{2}}\right),
$$

where $\theta_{\eta}$ are the nuisance parameters and $x_{i}$ and $\lambda_{i}$ the real and expected number of events in the $i$-th zenith-energy bin. The error in the nuisance parameters is assumed Gaussian with central value $\Theta_{\eta}$ and error $\sigma_{\eta}$. The expected number of events is computed propagating the neutrino fluxes with the numerical code [10] and using the detector montecarlo simulation released by the collaboration [7]. Details on the robustness of the result for different discrete systematic errors such as primordial fluxes, hadronic models and cross sections can be found in [1].

\section{Results}

The results are summarized by the posterior probabilities shown in fig. 2. On the left, we depict the one-dimensional probabilities for derived parameters such as the total mass, the mass of the core, the momentum of inertia, and the difference between the average density in the core and the mantle regions. On the right, we show the Earth's density distribution (68\% credible regions), reconstructed with one year of IceCube data (in blue).

IceCube has already collected more years of data and it may be interesting to see what can be done in the near future. To illustrate this in fig. 2 (right) we show in red the $68 \%$ regions for a 10-year forecast where mock data is generated assuming the PREM model and the central value for all the systematic errors. The treatment of the errors is the same as used in the one-year analysis.

\section{Acknowledgments}

This work is supported by the grant FPA2016-76005-C2-1-P, María de Maetzu grant MDM2014-0367, research grant 2017-SGR-929 and the European Horizon 2020 No. 690575 and 674896. 

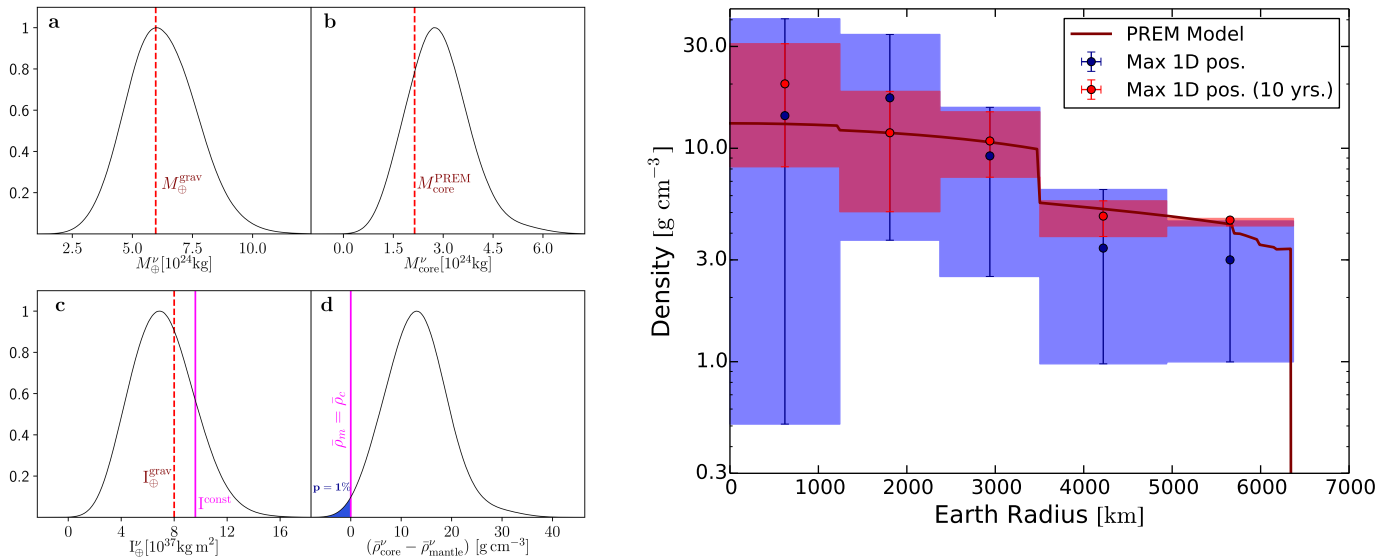

Figure 2: (left) Posterior 1D probabilities for derived parameters. (right) $68 \%$ probability regions for the different layers. In blue one year of data and in red 10-year forecast. From ref. [1]

\section{References}

[1] A. Donini, S. Palomares-Ruiz and J. Salvado, Neutrino tomography of Earth, Nature Phys. 15 (2019) no.1, 37 doi:10.1038/s41567-018-0319-1 [arXiv:1803.05901 [hep-ph]].

[2] B. A. Bolt, The precision of density estimation deep in the Earth, Q. J. R. Astron. Soc. 32, 367-388, year, 1991.

[3] W. Winter, Neutrino tomography: Learning about the earth's interior using the propagation of neutrinos, Earth Moon Planets 99 (2006) 285 doi:10.1007/s11038-006-9101-y [physics/0602049].

[4] A. Placci, E. Zavattini, On the possibility of using high-energy neutrinos to study the Earth's interior CERN Report 1973.

[5] L. V. Volkova and G. T. Zatsepin, On the problem of neutrino penetration though the earth. (talk, in russian), Izv. Akad. Nauk Ser. Fiz. 38N5 (1974) 1060.

[6] M. C. Gonzalez-Garcia, F. Halzen, M. Maltoni and H. K. M. Tanaka, Radiography of earth's core and mantle with atmospheric neutrinos, Phys. Rev. Lett. 100 (2008) 061802 doi:10.1103/PhysRevLett.100.061802 [arXiv:0711.0745 [hep-ph]].

[7] M. G. Aartsen et al. [IceCube Collaboration], Searches for Sterile Neutrinos with the IceCube Detector, Phys. Rev. Lett. 117 (2016) no.7, 071801 doi:10.1103/PhysRevLett.117.071801 [arXiv:1605.01990 [hep-ex]].

[8] K, Hoshina, H. K. M. Tanaka, Neutrino radiography with IceCube neutrino observatory, Poster at the XXV International Conference on Neutrino Physics and Astrophysics, June 3-9, 2012, Kyoto (Japan) 2012.

[9] Feroz, F. \& Hobson, M. P. Multimodal nested sampling: an efficient and robust alternative to MCMC methods for astronomical data analysis. Mon. Not. Roy. Astron. Soc. 384, 449 (2008).

[10] Argüelles Delgado, C. A., Salvado, J. \& Weaver, C. N. A simple quantum integro-differential solver (SQuIDS). Comput. Phys. Commun. 196, 569-591 (2015). 1412.3832. 